Highly Energetic Physical Processes and

Mechanisms for Emission from Astrophysical Plasmas

IAU Symposium, Vol. 195, 2000

P. C. H. Martens, S. Tsuruta, and M. A. Weber, eds.

\title{
Particle Acceleration in Solar Flares and Coronal Mass Ejections
}

\author{
R. P. Lin \\ University of California, Physics Department and Space Sciences \\ Laboratory, Berkeley, CA 94720, U.S.A.
}

\begin{abstract}
.
The Sun accelerates ions up to tens of $\mathrm{GeV}$ and electrons up to 100 s of $\mathrm{MeV}$ in solar flares and coronal mass ejections. The energy in the accelerated tens-of-keV electrons and possibly $\sim 1 \mathrm{MeV}$ ions constitutes a significant fraction of the total energy released in a flare, implying that the particle acceleration and flare energy release mechanisms are intimately related. The total rate of energy release in transients from flares down to microflares/nanoflares may be significant for heating the active solar corona.

Shock waves driven by fast CMEs appear to accelerate the highenergy particles in large solar energetic particle events detected at $1 \mathrm{AU}$. Smaller SEP events are dominated by $\sim 1$ to tens-of-keV electrons, with low fluxes of up to a few $\mathrm{MeV} /$ nucleon ions, typically enriched in ${ }^{3} \mathrm{He}$. The acceleration in gamma-ray flares appears to resemble that in these small electron $-{ }^{3} \mathrm{He}$ SEP events.
\end{abstract}

\section{Introduction}

The Sun is the most powerful natural particle accelerator in our solar system, able to accelerate ions to energies of tens of $\mathrm{GeV}$ and electrons to hundreds of $\mathrm{MeV}$. This acceleration occurs as a consequence of transient releases of energy in solar flares and coronal mass ejections (CMEs). Solar flares are the most powerful explosions in the solar system, able to release up to $10^{32}-10^{33}$ ergs in $10^{2}-10^{3} \mathrm{~s}$. The accelerated $10-100 \mathrm{keV}$ electrons (and possibly $\geq 1 \mathrm{MeV} /$ nucleon ions) appear to contain a significant fraction, perhaps the bulk, of this energy, indicating that the particle acceleration and energy release processes are intimately linked. How the Sun releases this energy, presumably stored in the magnetic fields of the corona, and how it rapidly accelerates electrons and ions with such high efficiency and to such high energies; is presently unknown. Furthermore, transient energy releases by the Sun extend down in size to at least microflares and perhaps even nanoflares $\left(\sim 10^{-6}\right.$ and $10^{-9}$, respectively, of the energy release of the largest flare). The total rate of energy release by all these flares and flare-like transients may be significant for the heating of the active corona, which is the dominant source of the soft X-ray luminosity of the Sun.

CMEs are transient ejections of material from the solar corona (the outer atmosphere of the Sun) into the interplanetary medium. On average, $\sim 5 \times 10^{15} \mathrm{~g}$ 
of material are ejected per CME, with a range of speeds from $\$ 100 \mathrm{~km} / \mathrm{s}$ to $\sim 2000 \mathrm{~km} / \mathrm{s}$. About $1 / 3$ of all CMEs are traveling fast enough, relative to the ambient medium, to drive a collisionless shock wave. These shocks appear to be responsible for accelerating the very high energy particles observed at $1 \mathrm{AU}$ in Large Solar Energetic Particles (LSEP) events. Smaller SEP events detected in the interplanetary medium are dominated by nonrelativistic, $\sim 1$ to tens-of-keV electrons, and appear to come from solar flares or flare-like events rather than CMEs. The ion components of these events are weak and usually only extend up to a few-MeV/nucleon energies, but often are highly enriched in ${ }^{3} \mathrm{He}$ and heavy elements.

The ability to release energy impulsively and accelerate particles to high energies is a common characteristic of magnetized cosmic plasmas at many sites throughout the universe, ranging from magnetospheres to active galaxies. The basic physics of these processes can best be studied on the active Sun; the accelerated particles range up to cosmic-ray energies, and the escaping particles can be sampled directly, while those which interact with the solar atmosphere can be observed via their electromagnetic emissions. Furthermore, the proximity of the Sun means the region where the flare energy release and particle acceleration takes place can be located, and the conditions within the region can be studied in detail to provide an understanding of these fundamental physical processes.
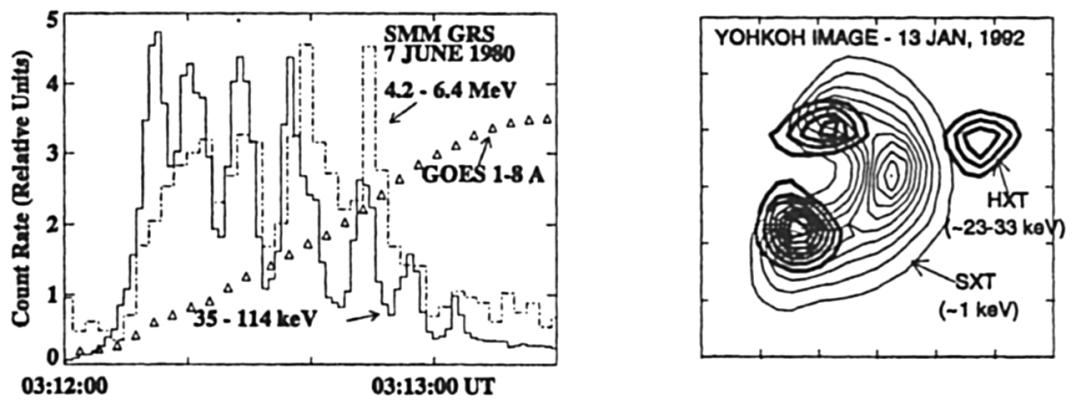

Figure 1. (a-left) Time profiles for a flare showing near-coincident impulsive peaks in 35-114 keV hard X-rays from energetic electrons and 4.2-6.4 MeV $\gamma$-rays from energetic ions (after Chupp 1990). (b-right) The M1 limb-flare seen with Yohkoh SXT and HXT (after Masuda et al. 1994).

\section{Solar Flares}

Bursts of hard X-rays ( $~ Z 20 \mathrm{keV})$ are the most common signature of the impulsive phase of a solar flare (Figure 1a). These X-rays are produced by bremsstrahlung of accelerated electrons colliding with the ambient solar atmosphere. If these electrons are indeed nonthermal (i.e., electron energy $E_{e} \gg k T$ of the ambient gas), then the energy lost by the electrons to bremsstrahlung in collisions with ambient ions is only a small fraction $\left(\sim 10^{-5}\right)$ of their energy lost to Coulomb collisions with ambient thermal electrons. This inefficiency means that, for many 
flares, the energy in accelerated $>20 \mathrm{keV}$ electrons must be comparable to the total flare radiative and mechanical output (Lin \& Hudson 1976). Thus, the acceleration of electrons to tens of $\mathrm{keV}$ may be the most direct consequence of the basic flare-energy release process.

During the flare's impulsive phase, the Yohkoh Hard X-ray Telescope (HXT) often observes double footpoint structures at tens of $\mathrm{keV}$ energies (Figure 1b), with the two footpoints brightening simultaneously to within a fraction of a second (Sakao 1994). These coincide, spatially and temporally, with $\mathrm{H} \alpha$ and white-light brightenings, implying that nonthermal, tens-of-keV electrons are interacting with a cool $\left(T<10^{5} \mathrm{~K}\right)$ environment. Very rapid transport of energy from the release site down to the footpoint interaction regions is required. This can only be achieved by fast electrons streaming down the loop and depositing their energy in lower coronal and chromospheric footpoints. The resulting heating of the ambient gas leads to evaporation of chromospheric material and flare radiation in the visible, UV/EUV, and soft X-rays (Figure 1a, triangles). Hard X-ray studies of electron time-of-flight (Aschwanden et al. 1996) further support this nonthermal, thick-target model, but it is unclear how the implied huge currents (up to $10^{36}$ electrons/s or $10^{17} \mathrm{amps}$ ) can conform to the global electrodynamic constraints of the flare environment (Miller et al. 1997).

For some flares occurring near the solar limb, HXT has detected a cotemporal, weaker, hard X-ray source in the corona (Masuda et al. 1994; Alexander \& Metcalf 1997) above the soft X-ray loop linking the hard X-ray footpoints (Figure 1b). This source has been interpreted as evidence for energy release by magnetic reconnection in a region above the soft $\mathrm{X}$-ray loop. Further evidence for this interpretation is provided by the observation of cusp-like structures in soft X-rays (Tsuneta 1996).

Hard (>20 keV) X-ray microflares, 10-100 times less intense than small flares, occur every $\sim 5$ minutes (Lin et al. 1984) near solar maximum, with the smaller ones occurring more frequently. Recently, high-sensitivity measurements down to $\sim 8 \mathrm{keV}$ with the Compton Gamma-Ray Observatory (CGRO) BATSE experiment show that solar impulsive bursts are observed $z 3$ times more often above $8 \mathrm{keV}$ than above $25 \mathrm{keV}$ (Feffer 1996; Lin 1997). Essentially every active-region transient brightening in thermal soft X-rays (Shimizu et al. 1995) is accompanied by an impulsive, nonthermal, $>8 \mathrm{keV}$ burst. This suggests that the flare process may be a fundamental way by which stored magnetic energy is transiently released in the Sun's corona (see Parker 1988). If the nonthermal spectra extend down to $\sim 5 \mathrm{keV}$, the average rate of energy deposition by microflare accelerated electrons, assuming nonthermal X-ray production, could be a significant fraction of that required to heat the active-region corona.

Nuclear collisions of accelerated protons, $\alpha$-particles, and heavier nuclei with the ambient solar atmosphere result in a rich spectrum (Figure 2) of lines (Ramaty \& Murphy 1987; see also Ramaty \& Mandzhavidze 2000, these Proceedings). Gamma-ray line emission has been observed from many solar flares, mostly by the Solar Maximum Mission (SMM) and CGRO (Chupp 1990; Share $\&$ Murphy 1995; Murphy et al. 1997). Energetic protons and $\alpha$-particles colliding with carbon and heavier nuclei produce narrow de-excitation lines (widths of $\sim$ few $\mathrm{keV}$ to $\sim 100 \mathrm{keV}$ ), while energetic heavy nuclei colliding with ambient hydrogen and helium produce much broader lines (widths of a few hundreds $\mathrm{keV}$ 


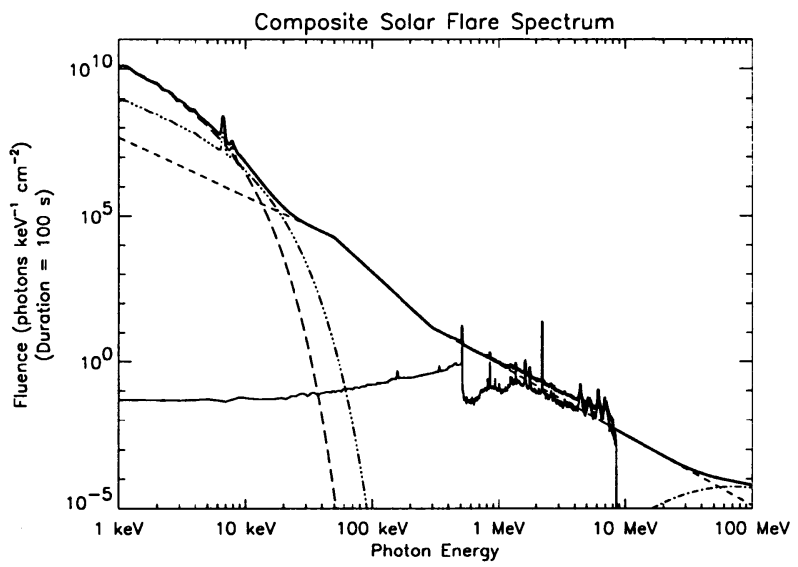

Figure 2. The composite spectrum (Dennis 1999) for a large flare, from multitemperature thermal flare plasma (dot-dash and long dash lines on left) through the hard X-ray/gamma-ray continuum (short dash and solid lines), gamma-ray lines (lower solid line), and pion decay (dot-dash line in lower right).

to an $\mathrm{MeV}$ ). Neutron capture on hydrogen and positron annihilation produce narrow lines (at $2.223 \mathrm{MeV}$ and $0.511 \mathrm{MeV}$, respectively) which are delayed.

In Figure 1a, the 4.1-6.4 MeV gamma-ray emission, which is dominated by broad and narrow lines produced by nuclear collisions of $z 10 \mathrm{MeV}$ ions, has a similar profile to the hard X-rays but with a delay of about 2 seconds. Thus, electrons and ions appear to be accelerated to high energies nearly simultaneously, on short time scales. Gamma-ray and neutron emission at tens to hundreds of $\mathrm{MeV}$, observed in very large flares, have similar time scales indicating that ions and electrons of hundreds of $\mathrm{MeV}$ to $\mathrm{GeV}$ energies are also accelerated rapidly.

The gamma-ray line observations show that $>10 \mathrm{MeV}$ ions are accelerated in essentially all large flares with intense hard X-ray emission but, because of the relatively low sensitivity of the gamma-ray observations, it is not known whether all flares accelerate $z 10 \mathrm{MeV}$ ions. The $10-100 \mathrm{MeV} /$ nucleon ions that produce the bulk of the $\gamma$-ray line emission contain only a small fraction of the energy in the $z 20 \mathrm{keV}$ electrons. However, systematic study of SMM $\gamma$-ray-line flares (Share \& Murphy 1995) shows that the $1.634 \mathrm{MeV}^{20} \mathrm{Ne}$ line is unexpectedly enhanced. Because the cross section for ${ }^{20} \mathrm{Ne}$ has an unusually low energy threshold $(\sim 2.5 \mathrm{MeV})$, this effect may be due to large fluxes of low-energy ions with a total energy content perhaps comparable to that in the accelerated electrons, rather than to an overabundance of neon (see Ramaty \& Mandzhavidze 2000, these Proceedings; Ramaty et al. 1995; Emslie, Brown, \& MacKinnon 1997).

\section{Large Solar Energetic Particle Events (LSEPs)}

Studies of solar energetic particles in the interplanetary medium (IPM) indicate that there are two main classes of events (Table 1). LSEP events produce a sig- 
Table 1. Solar Energetic Particle Event Characteristics

\begin{tabular}{|c|c|c|}
\hline Characteristic & $\begin{array}{l}\text { Large Solar Energetic Par- } \\
\text { ticle (LSEP) Events }\end{array}$ & $\begin{array}{l}\text { Nonrelativistic }{ }^{3} \mathrm{He} \text {-rich } \\
\text { Events }\end{array}$ \\
\hline Dominant Particle Species & $z 10 \mathrm{MeV}$ protons & $\sim 2-100 \mathrm{keV}$ electrons \\
\hline Electron to Proton Ratio & small & large \\
\hline${ }^{3} \mathrm{He} /{ }^{4} \mathrm{He}$ Ratio & $\begin{array}{l}\text { "normal" solar } \\
\left(\sim 5 \times 10^{-4}\right)\end{array}$ & $\begin{array}{l}\sim 0.1-1\left(\sim 10^{2} \text { to } \gtrsim 10^{3}\right. \\
\text { times normal })\end{array}$ \\
\hline Heavy Nuclei & "normal" solar & $\begin{array}{l}\text { enhanced abundances } \\
\text { of } \mathrm{Fe}, \mathrm{Mg}, \mathrm{Si}, \mathrm{S}\end{array}$ \\
\hline Ionization States & $\begin{array}{l}\text { typical of } 1-2 \times 10^{6} \mathrm{~K} \\
\text { normal corona } \\
\left(\mathrm{e} . \mathrm{g} ., \mathrm{Fe}^{+13}\right)\end{array}$ & $\begin{array}{l}\text { highly stripped, } \\
\left(\text { e.g., } \mathrm{Fe}^{+20}\right) \text { typical } \\
\text { of } \sim 10^{7} \mathrm{~K} \text { plasma }\end{array}$ \\
\hline Extent in Solar Longitude & $\gtrsim 100$ degrees & tens of degrees \\
\hline $\begin{array}{l}\text { Event Rate } \\
\text { (at solar maximum) }\end{array}$ & tens per year & $\gtrsim 10^{3}$ per year \\
\hline Flare Association & $\begin{array}{l}\text { large solar flare } \\
\text { (but sometimes missing) }\end{array}$ & $\begin{array}{l}\text { mostly small flares but } \\
\text { often no flare }\end{array}$ \\
\hline Solar Soft X-ray Burst & $\begin{array}{l}\text { gradual, e-folding decay } \\
\text { times }>10 \text { minutes }\end{array}$ & $\begin{array}{l}\text { impulsive, e-folding decay } \\
\text { times }<10 \text { minutes }\end{array}$ \\
\hline $\begin{array}{l}\text { Interplanetary } \\
\text { Association }\end{array}$ & $\begin{array}{l}\text { Coronal Mass Ejection } \\
\text { (CME) with fast shock }\end{array}$ & $\begin{array}{l}\text { interplanetary type III } \\
\text { radio burst }\end{array}$ \\
\hline
\end{tabular}

nificant flux of $z 10 \mathrm{MeV}$ protons (Figure 3a) and are the most intense and most energetic events. They usually occur after a large solar flare and occasionally exhibit acceleration up to relativistic energies, as in the first LSEP event discovered by Forbush (1946). Besides energetic ions, electrons are also observed, but the fluxes of energetic protons dominate over electrons. Tens of LSEP events are detected per year near solar maximum.

The solar energetic particles propagate away from the Sun along the Archimedean-spiral interplanetary magnetic field (Figure $3 \mathrm{~b}$ ). For magnetically wellconnected LSEP events, the energetic particle fluxes typically rise rapidly soon after a large solar flare (Figure 3a), with the fastest particles arriving earliest. The observed velocity dispersion in the onset times is usually consistent with particles of all energies being accelerated simultaneously near the Sun and travelling the same distance along the spiral interplanetary magnetic field. The rapid rise to maximum is usually followed by a much slower decay which has been attributed to scattering of the particles by irregularities in the interplanetary magnetic field and/or to prolonged acceleration.

The observed ionization states of LSEP particles are typical of a $1-2 \times 10^{6} \mathrm{~K}$ plasma, indicating they come from the quiescent solar corona and not from the hot, $Z 10^{7} \mathrm{~K}$, solar-flare plasma, from the solar photosphere, or from the chromosphere where temperatures are less than $10^{5} \mathrm{~K}$. The elemental composition of 


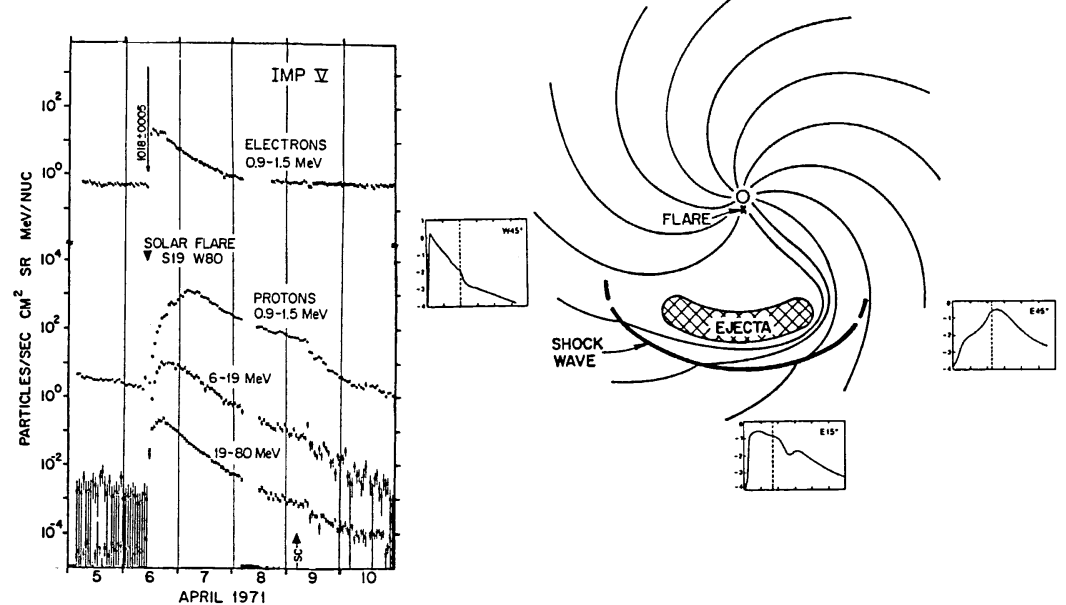

Figure 3. (a-left) Example of a Large Solar Energetic Particle (LSEP) event from a solar flare at $\mathrm{W} 80^{\circ}$ solar longitude (van Hollebeke, Wang, \& McDonald 1974). The passage of a shock wave is indicated by the sudden commencement (labeled SC at lower right) seen at the Earth. (b-right) The interplanetary magnetic field is drawn out to an Archimedes spiral as shown in the upper half. In the lower half, the rectangles show representative profiles of $20 \mathrm{MeV}$ protons seen by observers when the source flare is at various solar longitudes, assuming a fast coronal mass ejection (CME) is propagating outward with a shock wave (heavy line) ahead (adapted from Cane et al. 1988). For each profile, the dashed line indicates the time of passage of the CME shock wave. The vertical axis is the logarithm (base 10) of the proton flux, while each tick of the horizontal time scale is one day.

the LSEP particles, once they have been corrected for the $q / m$ (charge to mass) dependence of the acceleration and propagation processes, appear to closely reflect typical coronal abundances.

LSEP particles are often spread over about 100 degrees in solar longitude away from the flare site (Figure $3 \mathrm{~b}$ ). The observed lack of compositional variation with longitude, and the rapid onsets for LSEP events even for flares located far from the connection longitude, suggests that the acceleration itself is widespread, as expected for acceleration by widespread shock waves (see review by Lin, 1987). Since LSEP events and fast CMEs are typically accompanied by flare soft X-ray emission of relatively long duration, with e-folding decay times of $\gtrsim$ tens of minutes, LSEPs are also called "gradual" events.

Because of the scattering in the IPM, the time of acceleration near the Sun is difficult to determine accurately, but the LSEP energetic ions appear to be accelerated tens of minutes after the flare (Figure 4a), even after first-order corrections for scattering are taken into account (Kahler 1994). The delays are interpreted as evidence for acceleration of the ions by the CME shock when it reaches altitudes of $\sim 5$ to 15 solar radii. In LSEP-type events, the electrons also appear to be injected tens of minutes after the flare, especially in events 
which were not magnetically well connected (Krucker et al. 1999). For these delayed events, the EUV Imaging Telescope (EIT) on the Solar and Heliospheric Observatory (SOHO) often detects a flare wave propagating across the Sun at speeds of $\sim$ hundreds of $\mathrm{km} / \mathrm{s}$. The delays appear consistent with acceleration of the electrons by the wave/shock as it crosses the magnetic field line connected to the spacecraft at $1 \mathrm{AU}$.

An increase in the energetic ion fluxes, particularly at low $(\lesssim 1 \mathrm{MeV})$ energies, is often observed around the time of passage of a fast CME shock past the spacecraft, indicating that the shock can continue to accelerate particles even in the IPM near $1 \mathrm{AU}$. At late times in LSEP events, the shape of the time profile of the particle fluxes varies with solar longitude of the flare (Figure $3 b$ ) in a way that is consistent with distortion of the interplanetary magnetic structure by the CME and with continued acceleration by the shock wave as the CME and shock propagate outward through the IPM (Cane, Reames, \& von Rosenvinge 1988). Above several $\mathrm{MeV}$ energies, however, interplanetary shock acceleration effects are rarely, if ever, detected unless energetic particles are already present in the IPM. One of the primary unanswered questions for LSEP particle acceleration is: how are particles accelerated near the Sun by CME shocks to very high (GeV) energies?

\section{Solar, Nonrelativistic Electron- ${ }^{3}$ He-rich Events}

The second type of solar energetic particle event (Table 1) is dominated by electrons (Figure $4 \mathrm{~b}$ ) of $\sim 1$ to tens-of-keV energies. Such nonrelativistic electron events occurred far more frequently, $\sim 10^{3}$ events per year over the whole Sun at solar maximum, than the LSEP events (Lin 1985). Recent measurements show that the electron spectrum often continues to monotonically increase down to $\sim 1 \mathrm{keV}$ (Lin et al. 1996); many events are only detected below $\sim 10 \mathrm{keV}$. At these low energies, the electrons must have been accelerated high in the solar corona or else they would be lost to Coulomb collisions before escaping the Sun. As the electrons escape the Sun and travel outward through the IPM, they excite plasma waves which in turn produce solar type-III radio bursts.

In contrast to LSEP events, the electron-to-ion flux ratio is generally very large in nonrelativistic electron events. The ion fluxes are usually very low and dominated by low energy, $\lesssim 1 \mathrm{MeV} /$ nucleon ions. ${ }^{3} \mathrm{He}$ is often enriched over ${ }^{4} \mathrm{He}$ by factors of hundreds to thousands over the normal solar abundance ratio of $\sim 5 \times 10^{-4}$. Often protons, as well as ${ }^{4} \mathrm{He}$, were also observed to be very underabundant. As experiments improved in sensitivity, ${ }^{3} \mathrm{He}$-rich emission was observed to accompany essentially all of the more intense, nonrelativistic electron events (see Figure $4 \mathrm{~b}$ ).

The nonrelativistic electron- ${ }^{3} \mathrm{He}$ events are typically associated with small flares or subflares, or lack a flare entirely, and there is no association with CMEs. The electrons and ${ }^{3} \mathrm{He}$ nuclei often propagate nearly scatter-free through the IPM and quickly escape the inner solar system, leading to relatively rapid decay of the fluxes. Analysis of the velocity dispersion in the onset times indicates that the accelerations of the ${ }^{3} \mathrm{He}$ ions and electrons occur nearly simultaneously. The associated flares, when present, generally have impulsive, short duration (e-folding decay times $<10$ minutes) soft $\mathrm{X}$-ray bursts, so these are also called 

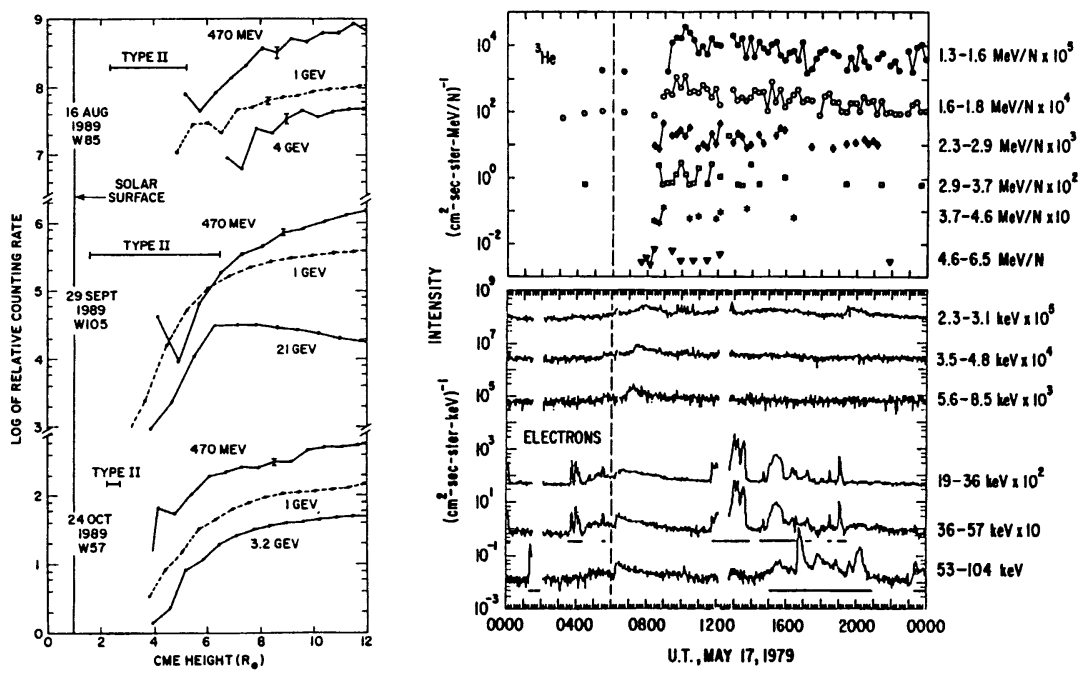

Figure 4. (a-left) Proton injection profiles near the Sun, inferred from the onsets for three LSEP events with ion emission, plotted versus the location of the associated CME. The injections appear to occur when the associated CME is at $z^{5-15}$ solar radii (from Kahler 1994). (b-right) Nonrelativistic electron- ${ }^{3} \mathrm{He}$-rich event (Reames, von Rosenvinge, \& Lin 1985). The lower panel shows an electron event beginning at $\sim 0620 \mathrm{UT}$ at $53-104 \mathrm{keV}$. Velocity dispersion is clearly evident with the lowest energy (2.3-3.1 keV) electrons beginning $\sim 0730$ UT. The horizontal lines indicate times of contamination by terrestrial, upstream particles or solar X-rays. The upper panel shows the associated ${ }^{3} \mathrm{He}$ event, which also exhibits velocity dispersion. In this event, the ${ }^{3} \mathrm{He} /{ }^{4} \mathrm{He}$ ratio is $>10$ and no solar flare was observed. The vertical dashed line indicates the time of the solar type-III radio burst. 
"impulsive" events. The flares are located near $\mathrm{W} 60^{\circ}$ longitude, i.e., magnetically well-connected to the terrestrial observer, with a spread of only a few tens of degrees in longitude. The smaller spread indicates that the acceleration process is probably localized in the vicinity of the flare.

The elemental composition in ${ }^{3} \mathrm{He}$-rich events shows substantial enhancement in the abundances of heavy nuclei; $\mathrm{Mg}, \mathrm{Si}, \mathrm{S}, \mathrm{Ne}$, and $\mathrm{Fe}$, but not $\mathrm{O}, \mathrm{C}$, or N (Reames, Meyer, \& von Rosenvinge 1994). The ionization states appear to be higher than for LSEPs; in early measurements, Fe was detected with an average charge state of around +20 for ${ }^{3} \mathrm{He}$-rich events compared to around +13 for LSEPs, suggesting that either the source has a temperature of $z 10^{7} \mathrm{~K}$, comparable to the heated flare plasma, or perhaps that the accelerated ions had undergone some stripping of their bound electrons. More recent charge-state measurements with the recently launched ACE spacecraft, however, do not appear to cleanly separate into these two classes.

The characteristics of nonrelativistic electron $-{ }^{3} \mathrm{He}$-rich events (Table 1 ) indicate that they comprise a class of solar particle acceleration events distinct from LSEPs. The enormous enhancement of ${ }^{3} \mathrm{He}$ suggests some sort of resonant acceleration process. An attractive theory has been suggested in analogy with phenomena observed in the Earth's auroral zone (Temerin \& Roth 1992). There, electromagnetic ion-cyclotron waves are often detected that have been driven unstable by auroral electron beams, which may be analogous to the electrons accelerated in solar impulsive events. For plasmas with solar composition, these waves have a frequency between the hydrogen gyrofrequency and the twoion hybrid frequency, slightly above the ${ }^{4} \mathrm{He}$ gyrofrequency. ${ }^{3} \mathrm{He}^{++}$, then, is the only ion which can be directly resonant with these waves, and it can be efficiently accelerated up to $\sim \mathrm{MeV} /$ nucleon energies.

\section{Discussion}

How are the energetic particle populations at the Sun, inferred from X-ray and gamma-ray emissions, related to the ones detected in SEPs in the IPM? Where both a flare hard X-ray burst and associated interplanetary electron event are observed, the number of electrons escaping to the interplanetary medium always appears to be only $0.1-1 \%$ of the number at the Sun. The sensitivity of the hard $\mathrm{X}$-ray observations is limited, however.

The ratio of the number of escaping $z 10 \mathrm{MeV}$ protons to the number of protons interacting to produce gamma-rays at the Sun varies from $\lesssim 10^{-3}$ to $\gtrsim 10^{2}$ (Cliver et al. 1989). The observed gamma-ray flares generally have small ratios of escaping-to-interacting ions, while many LSEP events have only upper limits on the gamma-ray line emission. The largest nuclear gamma-ray flares require the acceleration of about the same number of energetic $>10 \mathrm{MeV}$ ions as the largest LSEP events, to within an order of magnitude. Observations of nuclear gamma-ray lines show that $>10 \mathrm{MeV}$ ions are accelerated in essentially all large flares with intense hard X-ray emission, but it is not known whether small flares accelerate $z 10 \mathrm{MeV}$ ions. The high ambient density required for detectable gamma-ray line production implies that the gamma-ray-producing ions are not accelerated by CME shocks. 
The ratio of energetic electrons to energetic protons for nuclear gamma-ray flares, obtained through comparisons of the gamma-ray line emission to electron bremsstrahlung continuum emission, is substantially larger than observed for LSEP events, suggesting that nuclear gamma-ray flares may be more similar to nonrelativistic electron- ${ }^{3} \mathrm{He}$-rich events than to LSEP events (see Table 1). The soft X-ray bursts for these flares can be either impulsive or gradual. Recent analysis of the Doppler-broadened nuclear gamma-ray line spectra from SMM and CGRO (Share \& Murphy 1999) have shown that accelerated ${ }^{56} \mathrm{Fe}$ is enhanced over ambient $\mathrm{Fe}$ by about an order of magnitude, consistent with electron- ${ }^{3} \mathrm{He}$-rich events, but the enhancements of accelerated $\mathrm{Mg}$ and $\mathrm{Ne}$ over their ambient values are significantly lower than in those events.

The acceleration process in flares, particularly for nonrelativistic electrons, appears to be intimately related to the basic energy release process in flares, but the actual acceleration mechanism remains a mystery. High spectral-resolution hard X-ray observations of a flare show a sharp break (Lin \& Schwartz 1987) which suggests field-aligned, DC electric fields (Johns \& Lin 1992; Lin \& Johns 1993; Holman 1995) are at least partly responsible, but stochastic acceleration by waves (Miller 1991) may also be important. Planned future spacecraft missions, such as the High Energy Solar Spectroscopic Imager (HESSI) scheduled for launch in mid-2000, and the Particle Acceleration Solar Observatory (PASO) planned to be synchronously orbiting at $0.17 \mathrm{AU}$ (for the following solar maximum) may provide the key observations required for understanding of the physics of these fundamental processes.

Acknowledgments. This research was supported in part by NASA Contract NAS5-98033 and NASA Grant 5-6928.

\section{References}

Alexander, D., \& Metcalf, T. 1997, ApJ, 489, 442

Aschwanden, M. J., Hudson, H., Kosugi, T., \& Schwartz, R. A. 1996, ApJ, 464, 985

Cane, H. V., Reames, D. V., \& von Rosenvinge, T. T. 1988, J. Geophys. Res., 93,9555

Chupp, E. L. 1982, in AIP Conf. Proc., 77, Gamma Ray Transients and Related Astrophysical Phenomena, eds. R. E. Lingenfelter, H. S. Hudson, \& D. M. Worrall (New York: AIP), 363

Chupp, E. L. 1990, Physica Scripta, T18, 15

Cliver, E. W., Forrest, D. J., Cane, H. V., Reames, D. V., McGuire, R. E., von Rosenvinge, T. T., Kane, S. R., \& MacDowall, R. J. 1989, ApJ, 343, 953

Emslie, A. G., Brown, J. C., \& MacKinnon, A. L. 1997, ApJ, 485, 430

Feffer, P. T. 1996, Ph.D. thesis, University of California at Berkeley

Forbush, S. E. 1946, Phys. Rev., 70, 771

Holman, G. D. 1995, ApJ, 452, 451

Johns, C. M., \& Lin, R. P. 1992, Solar Phys., 137, 121

Kahler, S. 1994, ApJ, 428, 837 
Krucker, S., Larson, D. E., Lin, R. P., \& Thompson, B. J. 1999, ApJ, 519, 864 Lin, R. P. 1985, Solar Phys., 100, 537

Lin, R. P. 1987, Rev. Geophys., 25, 676

Lin, R. P. 1997, in AIP Conf. Proc., 385, Robotic Exploration Close to the Sun: Scientific Basis, ed. S. R. Habbal (New York, AIP), 25

Lin, R. P., \& Hudson, H. S. 1976, Solar Phys., 50, 153

Lin, R. P., \& Johns, C. M. 1993, ApJ, 417, L53

Lin, R. P., \& Schwartz, R. E. 1987, ApJ, 312, 462

Lin, R. P., Schwartz, R. A., Kane, S. R., Pelling, R. M., \& Hurley, K. C. 1984, ApJ, 283, 421

Lin, R. P., et al. 1996, Geophys. Res. Lett., 23, 1211

Mandzhavidze, N., \& Ramaty, R. 1993, Nucl. Phys. B (Proc. Suppl.), 33, 141

Masuda, S., Kosugi, T., Hara, H., Tsuneta, S., \& Ogawara, Y. 1994, Nature, 371,495

Miller, J. A. 1991, ApJ, 376, 342

Miller, J. A., et al. 1997, J. Geophys. Res., 102, 14631

Murphy, R. J., Share, G. H., Grove, J. E., Johnson, W. N., Kinzer, R. L., Kurfess, J. D., Strickman, M. S., \& Jung, G. V. 1997, ApJ, 490, 883

Parker, E. N. 1988, ApJ, 330, 474

Ramaty, R., \& Mandzhavidze, N. 2000, these Proceedings

Ramaty, R., Mandzhavidze, N., Kozlovsky, B., \& Murphy, R. 1995, ApJ, 455, L193

Ramaty, R., \& Murphy, R. J. 1987, Space Sci. Rev., 45, 213

Reames, D. V., Meyer, J. P., \& von Rosenvinge, T. T. 1994, ApJS, 90, 649

Reames, D. V., von Rosenvinge, T. T., \& Lin, R. P. 1985, ApJ, 292, 716

Sakao, T. 1994, Ph.D. Thesis, University of Tokyo

Share, G. H., \& Murphy, R. J. 1995, ApJ, 452, 933

Shimizu, T. 1995, PASJ, 47, 251

Temerin, M., \& Roth, I. 1992, ApJ, 391, L105

Tsuneta, S. 1996, ApJ, 456, 840

van Hollebeke, M. A. I., Wang, J. R., \& McDonald, F. B. 1974, Goddard Space Flight Center Publication, X-661-74-27 